\title{
Article \\ Circulating Forms of Urokinase-Type Plasminogen Activator Receptor in Plasma Can Predict Recurrence and Survival in Patients with Urothelial Carcinoma of the Bladder
}

\author{
Line H. Dohn ${ }^{1,2, *}$, Peter Thind ${ }^{3}$, Lisbeth Salling ${ }^{3}$, Henriette Lindberg ${ }^{4} \mathbb{D}$, Sofie Oersted ${ }^{1}$, Ib J. Christensen ${ }^{2,5,6}$, \\ Ole D. Laerum ${ }^{2,5}$, Martin Illemann ${ }^{2,5}$, Hans von der Maase ${ }^{1} \mathbb{D}$, Gunilla Høyer-Hansen ${ }^{2,5}$ (D) and Helle Pappot ${ }^{1} \mathbb{D}$
}

check for updates

Citation: Dohn, L.H.; Thind, P.; Salling, L.; Lindberg, H.; Oersted, S.; Christensen, I.J.; Laerum, O.D.; Illemann, M.; von der Maase, H.; Høyer-Hansen, G.; et al. Circulating Forms of Urokinase-Type

Plasminogen Activator Receptor in Plasma Can Predict Recurrence and Survival in Patients with Urothelial Carcinoma of the Bladder. Cancers 2021, 13, 2377. https://doi.org/ $10.3390 /$ cancers 13102377

Academic Editor: Andrea Morrione

Received: 16 March 2021

Accepted: 7 May 2021

Published: 14 May 2021

Publisher's Note: MDPI stays neutral with regard to jurisdictional claims in published maps and institutional affiliations.

Copyright: (c) 2021 by the authors. Licensee MDPI, Basel, Switzerland. This article is an open access article distributed under the terms and conditions of the Creative Commons Attribution (CC BY) license (https:// creativecommons.org/licenses/by/ $4.0 /)$.
1 Department of Oncology, Rigshospitalet, Blegdamsvej 9, 2100 Copenhagen, Denmark; sofie.oersted@regionh.dk (S.O.); hans@vondermaase.dk (H.v.d.M.); helle.pappot@regionh.dk (H.P.)

2 The Finsen Laboratory, Rigshospitalet, Copenhagen Biocenter, Ole Maaloes Vej 5, Building 3, 3rd Floor, 2200 Copenhagen, Denmark; ib.jarle.christensen@regionh.dk (I.J.C.); ole.larum@uib.no (O.D.L.); milleman@its.jnj.com (M.I.); gunillaogbent@hotmail.com (G.H.-H.)

3 Department of Urology, Rigshospitalet, Blegdamsvej 9, 2100 Copenhagen, Denmark; peter.ole.thind@regionh.dk (P.T.); lisbeth.Salling@regionh.dk (L.S.)

4 Department of Oncology, Herlev and Gentofte Hospital, Herlev, Borgmester Ib Juuls Vej 1, 2730 Herlev, Denmark; henriette.lindberg@regionh.dk

5 Biotech Research and Innovation Centre (BRIC), University of Copenhagen, Ole Maaloes Vej 5 , 2200 Copenhagen, Denmark

6 Department of Surgical Gastroenterology, Hvidovre Hospital, 2650 Hvidovre, Denmark

* Correspondence: line.hammer.dohn.02@regionh.dk; Tel.: +45-40783032

Simple Summary: Bladder cancer is an aggressive disease and after operation many patients are at risk of recurrence and shortened survival. Specific proteins are known to be of importance in the development of cancers. One of these proteins is the urokinase-type plasminogen activator receptor (uPAR) which exists in different forms. We here investigate the presence of the different uPAR forms in plasma from patients with bladder cancer, and we associate the elevated amount of uPAR forms with survival. We find that high levels of all uPAR forms is associated with short survival in patients with bladder cancer and suggest that this in the future might help improve handling of the disease.

Abstract: Urothelial carcinoma of the bladder is a highly aggressive disease characterised by a very heterogeneous clinical outcome. Despite cystectomy, patients still have a high recurrence risk and shortened survival. Urokinase-type plasminogen activator receptor (UPAR) is present in tumour tissue specimens from patients with urothelial carcinoma. The different UPAR forms in blood are strong prognostic markers in other cancer types. We investigate the presence of different uPAR forms in tumour tissue and test the hypothesis that preoperative plasma levels of the uPAR forms predict recurrence free survival, cancer specific survival, and overall survival in patients treated with cystectomy for urothelial carcinoma. Using Western blotting we analyse neoplasia and adjacent benign-appearing urothelium from randomly selected patients for the presence of intact and cleaved uPAR forms. Prospectively collected preoperative plasma samples from 107 patients who underwent radical cystectomy for urothelial carcinoma are analysed. The different uPAR forms are measured by time-resolved fluorescence immunoassays. UPAR in tumour tissue from patients with urothelial carcinoma is demonstrated in both an intact and cleaved form. The different uPAR forms in plasma are all significantly associated with both recurrence free survival, cancer specific survival, and overall survival, high concentrations predicting short survival. uPAR (I) has the strongest association with a HR of 2.56 for overall survival. In the multivariable survival analysis uPAR (I) is significantly associated with cancer specific survival and overall survival.

Keywords: bladder cancer; urothelial cancer; blood; plasma; urokinase receptor; uPAR; plasminogen activation; biomarker; survival; recurrence 


\section{Introduction}

For patients with high-risk non-muscle invasive and muscle invasive urothelial carcinoma of the bladder (UCB) without radiological signs of metastasis, radical cystectomy (RC) with lymphadenectomy is the standard, potentially curative, treatment [1,2]. RC affords effective local disease control; however, around $50 \%$ of patients with node negative disease will die of their cancer within five years [3,4]. Patients with histologically confirmed lymph node metastases have an even higher risk [3]. Neoadjuvant cisplatin-based chemotherapy is recommended in patients with muscle invasive disease without signs of lymph node metastases or distant metastasis, as it has shown an $8 \%$ absolute improvement in survival at five years [2,5]. Despite aggressive therapy, patients suffer from high rates of disease recurrence and shortened survival. Current tools, such as standard histopathologic risk factors and imaging are insufficient to detect micro-metastatic disease [6,7]. Hence, there is a strong need for additional biomarkers that at an early time-point can help capture the true clinical and biological potential of a tumour to permit a more individualised guidance for therapy or follow-up.

Detachments of cancer cells from the primary tumour, cancer cell migration and invasion into the surrounding tissue require degradation and remodelling of the extracellular matrix, which is mediated by a complex array of extracellular proteases [8]. The plasminogen activation system is one of the central protease systems involved in these processes. uPAR localises its ligand urokinase-type plasminogen activator (uPA) to the cell surface. Full length uPAR [uPAR (I-III)] consists of three domains and is attached to the cell membrane by a glycolipid anchor on domain III [9]. uPA bound to uPAR is capable of cleaving neighbouring cell-bound UPAR, liberating the UPAR (I) and leaving the cleaved form, uPAR (II-III), on the cell surface [9]. Therefore, the cleaved uPAR forms reflect the activity of the plasminogen activation system. uPAR (I-III) and UPAR (II-III) can be shed from the cell surface; consequently one intact and two cleaved UPAR forms are present in blood. Use of immunoassays measuring the individual forms of uPAR have demonstrated that the cleaved uPAR forms are strong prognostic biomarkers in other cancers, and better than the total amount of uPAR [10-12]. The cleaved uPAR forms measured in blood have not been investigated in UCB but we know from studies in tumour tissue from patients with UCB that a significant association exists between UPAR expression and increasing tumour stage, as well as an association between uPAR expression and poor survival [13-15]. The UPAR antibodies that work on paraffin embedded tissue are, however, not able to distinguish between different uPAR forms [9].

Taken all together there is an ongoing need to identify new selection markers which can be important tools to detect micro-metastatic disease- the earliest form of advanced bladder cancer. The UPAR forms measured in blood might be such new selection markers.

The aim of the study was to identify the presence of different uPAR forms in tissue and blood from UCB patients. We have used Western blotting of tumour tissue and timeresolved fluorescence immunoassays on plasma samples. In addition, we have tested the hypothesis that high preoperative plasma levels of the intact and cleaved UPAR forms can predict recurrence free survival, cancer specific survival, and overall survival in patients treated with RC for UCB.

\section{Materials and Methods}

\subsection{Tumour Tissue}

For Western blotting, fresh frozen muscle invasive tumour tissue as well as adjacent benign-appearing tissue from two patients-not included in the blood sample material below - treated with RC for UCB was analysed.

\subsection{Patients and Blood Samples}

Prospectively collected, preoperative EDTA plasma levels of the different uPAR forms were measured in 107 patients ( 20 women [19\%] and 87 men [ $81 \%$ ]; median age 67 years, range 44-81 at treatment start), who underwent RC with intended extended lymphadenec- 
tomy between March 2012 and April 2015 at the Department of Urology, Rigshospitalet, Copenhagen or the Department of Urology, Herlev Hospital, Denmark. The median number of lymph nodes resected was 18 (range 4-44). Six patients had $\leq 10$ lymph nodes removed. Eligible for inclusion were individuals with histopathological diagnosis of UCB with no evidence of other cancer within the last five years. Indications for RC were high-risk non-muscle invasive or muscle invasive disease without signs of lymph node metastases or distant metastasis, as investigated by computed tomography (CT) scan. Patients with T2-T4a were candidates for neoadjuvant chemotherapy. This supplementary treatment was, however, not applied for all study patients, as neoadjuvant chemotherapy was under implementation during the study period.

\subsection{Histopathology}

Histological features were collected from the histological reports. Pathologic tumour stage and grade were achieved using criteria from the UICC [16] and the WHO grading system [17]. Pathologic subgroups were defined as $\mathrm{pT} \leq \mathrm{pT} 2, \mathrm{pN} 0$ vs. $\mathrm{pT} \geq \mathrm{pT} 3$, $\mathrm{pN} 0$ vs. pTany, $\mathrm{pN}+$.

\subsection{Postoperative Follow-Up}

Follow-up schedule was performed according to institutional protocols. In general, patients with organ confined node negative disease ( $\mathrm{pT} \leq \mathrm{pT} 2$, $\mathrm{pN} 0$ ) were CT-scanned 6, 12 and 24 months postoperatively, while patients with non-organ confined disease ( $\mathrm{pT} \geq \mathrm{pT}$, pN0 or pTany, $\mathrm{pN}+$ ) were CT-scanned 6, 12, 18, 24 and 36 months after surgery.

Follow-up was defined as the interval from RC until death; if death had not occurred, data was censored at time of analysis (August 2017). The primary endpoint of this study was recurrence free survival (RFS) and the secondary endpoints were cancer specific survival (CSS) and overall survival (OS) [18]. Date of first recurrence, as well as date and cause of death, were obtained by retrospective chart review. Cancer detection in the ureter or urethra was coded as a second primary cancer and not as local or distant recurrence. Perioperative mortality (any death within 30 days of surgery) was censored at time of death for CSS analysis [18].

\subsection{Blood Samples}

Blood samples were collected prior to treatment start defined as the date of cystectomy or in patients receiving neoadjuvant chemotherapy as day 1 of chemotherapy. For preparation of EDTA plasma, blood samples were collected and handled as previously described [19].

\subsection{Measurements of uPAR Forms in Plasma}

Three different time-resolved fluorescence immunoassays (TR-FIAs) were used: TRFIA 1 measuring uPAR (I-III) [20], TR-FIA 2 measuring uPAR (I-III) + uPAR (II-III) [20], and TR-FIA 4 measuring uPAR (I) [10]. All three assays have been validated for use in $20 \%$ EDTA plasma $[19,21]$. Samples were measured in duplicate, and the mean values were used for statistical analyses. The inter-assay variability was below $10 \mathrm{CV} \%$. The $\mathrm{CV} \%$ for the duplicate measurements was below 20. The limit of quantification in 20\% EDTA plasma is for TR-FIA $10.8 \mathrm{pmol} / \mathrm{L}$, TR-FIA $22.3 \mathrm{pmol} / \mathrm{L}$ [21] and for TR-FIA $40.41 \mathrm{pmol} / \mathrm{L}$ [19]. The recovery of uPAR (I-III) in $20 \%$ EDTA plasma is $91 \%$ for TR-FIA 1, 95\% for TR-FIA 2 [21], and the recovery of UPAR (I) is $91 \%$ for TR-FIA 4 [19].

\subsection{Western Blot}

Frozen tissue was pulverised using a dry ice cooled tissue homogeniser. The tissue powder preparations were lysed using temperature induced phase separation in Triton X-114 containing buffer $(0.1 \mathrm{M}$ Tris/HCl, $\mathrm{pH}$ 8.1, 1\% Triton X-114, $10 \mathrm{mM}$ EDTA, $10 \mu \mathrm{g} / \mathrm{mL}$ aprotinin and $1 \mathrm{mM}$ phenyl-methyl-sulfonyl fluoride; $10 \mathrm{~mL}$ buffer/g frozen tissue) [22]. uPAR (I-III) and uPAR (II-III) separate to the detergent-phase because of 
their glycolipid anchor. The detergent-phases were chemically reduced, de-glycosylated using N-glycosidase F (Roche Diagnostic, Mannheim, Germany) and analysed by Western blotting using the monoclonal antibody S1 [23]. Detergent-phase from $2.5 \mathrm{mg}$ tissue was loaded in each lane. Detergent-phase from $5 \times 10^{5}$ cells of the human histiocytic lymphoma cell line U937 was loaded as control [23]. The proteins were separated on 4-12\% SDS-PAGE and were electroblotted onto polyvinylidene difluoride membranes using the iBlot system (Life Technologies) and Western blotting conducted essentially as described [24] using 2 $\mu \mathrm{g} / \mathrm{mL}$ of $\mathrm{S} 1$ and $1.3 \mu \mathrm{g} / \mathrm{mL}$ horseradish peroxidase conjugated rabbit anti-mouse IgG (P0161, Dako, Glostrup, Denmark).

\subsection{Statistics}

Descriptive statistics for continuous covariates are presented by the median as well as the minimum and maximum, and categorical variables by the frequencies. Spearman rank correlation was used as a measure of association between the UPAR levels and tests comparing levels between categories were done using the Wilcoxon rank sum test. Analyses of time to event data (RFS, CSS and OS) were performed using the Cox proportional hazards model. Results are presented by hazard ratios (HR) with 95\% confidence intervals (CI). Final multivariable models were identified using backwards selection. The concordance index as a measure of discrimination was calculated for each outcome [25]. The Cox models have been assessed based on martingale residuals. The levels of the different uPAR forms have been evaluated by the actual score on the log scale (base 2, implying that HRs are for a two-fold difference in levels) and dichotomised based on the 95th percentile from a reference set [19]. The level of significance was set to $5 \%$. All statistical calculations have been done using SAS (v9.4, SAS Institute, Cary, NC, USA) and R (R version 3.6.3).

\section{Results}

\subsection{Intact and Cleaved UPAR in UCB Tumour Tissue}

Western blot of tumour tissue samples shows two bands corresponding to the intact (uPAR (I-III)) (35 kDa), and cleaved uPAR (uPAR (II-III)) (27 kD) [9,23] (Figure 1). For the original Western blot figure see Figures S1 and S2). In agreement with our previous findings using immunohistochemistry $[13,14]$ the uPAR forms are highly up-regulated in the tumour tissue as compared to the adjacent benign-appearing tissue.

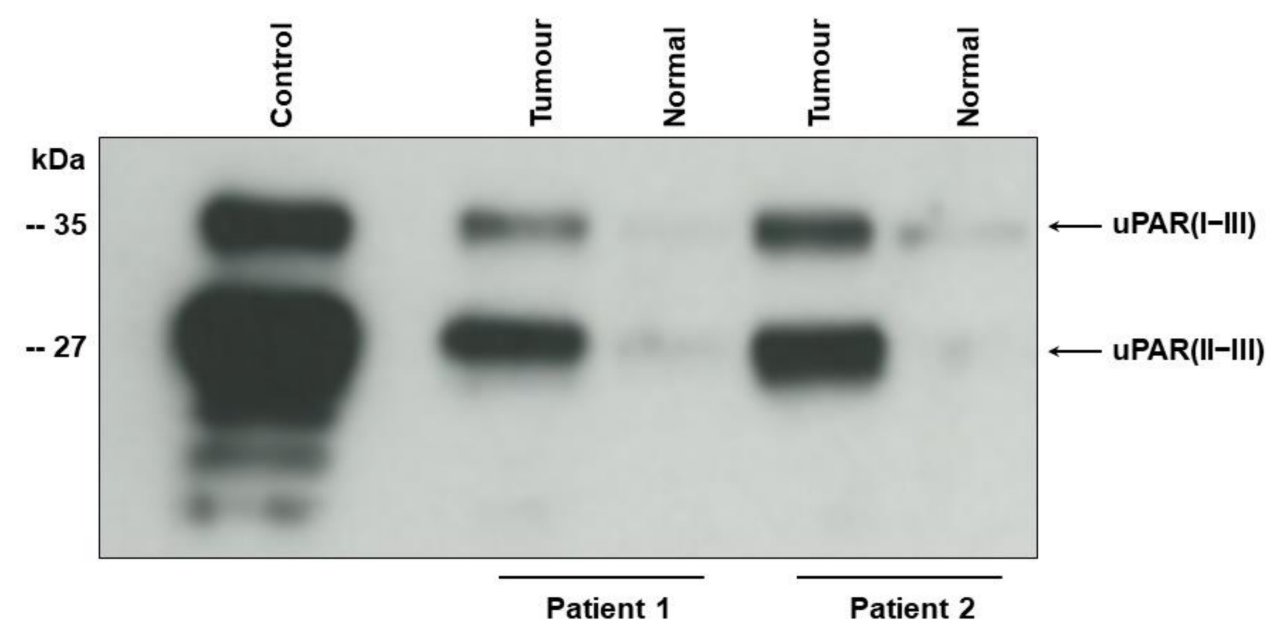

Figure 1. Intact and cleaved UPAR in tumour tissue from two patients with UCB.

Detergent-phase of UCB tissue (tumour) and adjacent benign appearing tissue (normal) was subjected to enzymatic de-glycosylation before electrophoresis to allow complete separation of uPAR (I-III) and UPAR (II-III). The monoclonal antibody S1 reacting with an epitope on domain II and III of chemically reduced UPAR [23] was used for Western 
blotting. Detergent-phase from U937 cells (control) was applied as positive control [23]. Electrophoretic mobility of standard proteins is indicated to the leftt.

\subsection{Association of Preoperative Plasma UPAR Forms with Clinical and Pathologic Characteristics}

The UPAR forms were measured in EDTA plasma samples from 107 patients. Patient characteristics are presented in Table 1.

Table 1. Patient characteristics $(n=107)$.

\begin{tabular}{|c|c|}
\hline Characteristics & $N(\%)$ \\
\hline \multicolumn{2}{|l|}{ Age } \\
\hline Years median (range) & $67(44-81)$ \\
\hline \multicolumn{2}{|l|}{ Gender } \\
\hline Female & $20(19)$ \\
\hline Male & $87(81)$ \\
\hline \multicolumn{2}{|c|}{ Pathologic stage (cystectomy specimen) } \\
\hline T0 & $36(34)$ \\
\hline CIS & $15(14)$ \\
\hline Тa & $4(4)$ \\
\hline $\mathrm{T} 1$ & $4(4)$ \\
\hline $\mathrm{T} 2$ & $22(20,5)$ \\
\hline $\mathrm{T} 3$ & $22(20,5)$ \\
\hline $\mathrm{T} 4$ & $4(4)$ \\
\hline \multicolumn{2}{|c|}{ Pathologic stage (cystectomy specimen) } \\
\hline $\mathrm{pT} \leq \mathrm{pT} 2 \mathrm{pN} 0$ & $76(71)$ \\
\hline $\mathrm{pT} \geq \mathrm{pT} 3 \mathrm{pN} 0$ & $17(16)$ \\
\hline pTany pN+ & $14(13)$ \\
\hline \multicolumn{2}{|c|}{ Pathologic grade (cystectomy specimen) } \\
\hline LG & $2(2)$ \\
\hline HG & $69(64)$ \\
\hline \multicolumn{2}{|l|}{ Concomitant CIS ${ }^{1}$} \\
\hline No & $65(61)$ \\
\hline Yes & $42(39)$ \\
\hline \multicolumn{2}{|l|}{ Lymph vascular invasion } \\
\hline No & $93(87)$ \\
\hline Yes & $14(13)$ \\
\hline \multicolumn{2}{|l|}{ Resection margin } \\
\hline No & $101(94)$ \\
\hline Yes & $6(6)$ \\
\hline \multicolumn{2}{|l|}{ Lymph node metastasis } \\
\hline No & $93(87)$ \\
\hline Yes & $14(13)$ \\
\hline \multicolumn{2}{|l|}{ Neoadjuvant chemotherapy } \\
\hline No & $79(74)$ \\
\hline Yes & $28(26)$ \\
\hline
\end{tabular}

${ }^{1}$ CIS = carcinoma in situ; LG = low grade; HG = high grade.

uPAR (I-III) was significantly associated with gender, uPAR (I-III) + uPAR (II-III) was significantly associated with gender, pathologic stage and resection margin, whereas uPAR(I) was significantly associated with gender and lymph node metastases. No association was found between plasma levels of any uPAR form and age, tumour grade, concomitant carcinoma in situ (CIS) or lymph vascular invasion. Correlations (rs) between uPAR (I-III) and uPAR (I-III) + uPAR (II-III), uPAR (I-III) and uPAR (I), and uPAR (I-III) + UPAR (II-III) and UPAR (I) were $0.74,0.44$ and 0.69 , respectively. Association 
of the preoperative plasma uPAR forms with clinical and pathological characteristics are presented in Table 2.

Table 2. Association of the preoperative plasma uPAR forms with clinical and pathological characteristics.

\begin{tabular}{|c|c|c|c|c|c|c|}
\hline \multirow[t]{3}{*}{ Characteristics } & \multicolumn{2}{|l|}{ uPAR (I-III) } & \multicolumn{2}{|c|}{ uPAR (I-III) + uPAR (II-III) } & \multicolumn{2}{|l|}{ uPAR (I) } \\
\hline & \multicolumn{2}{|l|}{$\mathrm{pmol} / \mathrm{L}$} & \multicolumn{2}{|l|}{$\mathrm{pmol} / \mathrm{L}$} & \multicolumn{2}{|l|}{$\mathrm{pmol} / \mathrm{L}$} \\
\hline & Median (Min-Max) & $p$-Value & Median (Min-Max) & $p$-Value & Median (Min-Max) & $p$-Value \\
\hline \multicolumn{7}{|l|}{ Age } \\
\hline $67(44-81)^{a}$ & 0.15 & 0.13 & 0.13 & 0.17 & -0.03 & 0.78 \\
\hline \multicolumn{7}{|l|}{ Gender } \\
\hline Female & 53.65 (31.98-91.43) & & $94.73(68.90-226.21)$ & & $38.85(17.04-754.88)$ & \\
\hline Male & $42.69(19.34-76.66)$ & 0.003 & 84.68 (51.60-156.67) & 0.02 & $35.60(16.40-124.15)$ & 0.05 \\
\hline \multicolumn{7}{|c|}{ Pathologic stage (cystectomy specimen) ${ }^{b}$} \\
\hline $\mathrm{pT} \leq \mathrm{pT} 2 \mathrm{pN} 0$ & $42.72(19.34-76.66)$ & & $85.14(51.60-156.67)$ & & 34.09 (16.40-124.15) & \\
\hline $\mathrm{pT} \geq \mathrm{pT} 3 \mathrm{pN} 0$ & $53.28(31.44-68.37)$ & 0.15 & 101.25 (73.52-145.19) & 0.03 & $42.99(17.04-86.66)$ & 0.06 \\
\hline pTany pN+ & 42.25 (27.47-91.43) & & $84.99(55.20-226.21)$ & & $34.00(21.08-754.88)$ & \\
\hline \multicolumn{7}{|l|}{ Pathologic grade } \\
\hline LG & $54.96(45.91-64.00)$ & & 107.33 (79.36-135.29) & & $58.14(57.62-58.65)$ & \\
\hline HG & $44.50(25.62-91.43)$ & 0.59 & $87.51(51.60-226.21)$ & 0.71 & $36.45(16.40-754.88)$ & 0.38 \\
\hline \multicolumn{7}{|l|}{ Concomitant CIS } \\
\hline No & 42.71 (19.34-91.43) & & 88.19 (53.07-226.21) & & $35.91(19.38-754.88)$ & \\
\hline Yes & 48.09 (26.49-76.66) & 0.39 & 89.56 (51.60-132.43) & 0.96 & $38.89(16.40-142.10)$ & 0.44 \\
\hline \multicolumn{7}{|c|}{ Lymph vascular invasion } \\
\hline No & 44.05 (19.34-91.43) & & 88.19 (51.60-156.67) & & $35.91(16.40-754.88)$ & \\
\hline Yes & $46.06(32.02-78.11)$ & 0.53 & $89.15(57.15-226.21)$ & 0.31 & $39.60(24.68-142.10)$ & 0.20 \\
\hline \multicolumn{7}{|l|}{ Resection margin } \\
\hline Negative & $43.23(19.34-91.43)$ & & $87.51(51.60-156.67)$ & & $35.91(16.40-754.88)$ & \\
\hline Positive & $51.12(44.41-78.11)$ & 0.09 & $128.56(77.47-226.21)$ & 0.05 & $46.75(25.00-107.60)$ & 0.27 \\
\hline \multicolumn{7}{|c|}{ Lymph node metastasis } \\
\hline No & $44.41(19.34-76.66)$ & & $89.35(51.60-156.67)$ & & $36.45(16.40-124.15)$ & \\
\hline Yes & $42.25(27.47-91.43)$ & 0.92 & $84.99(55.20-226.21)$ & 0.87 & $34.00(21.08-754.88)$ & 0.05 \\
\hline \multicolumn{7}{|c|}{ Neoadjuvant chemotherapy } \\
\hline No & $44.41(/ 19.34-78.11)$ & & $87.51(5160-228.21)$ & & $35.60(16.40-142.10)$ & \\
\hline Yes & $43.55(25.62-91.43)$ & 0.85 & $89.42(57.04-156.67)$ & 0.97 & $37.61(19.38-754.88)$ & 0.17 \\
\hline
\end{tabular}

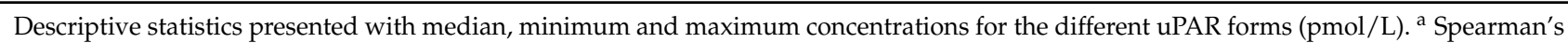
rank correlation coefficient ( $\mathrm{rs}$ ), $p$-value testing the hypothesis that $\mathrm{rs}=0 .{ }^{\mathrm{b}} p$-values are for the Wilcoxon rank sum test comparing the levels of the uPAR concentrations for baseline patients characteristic. CIS = carcinoma in situ; LG = low grade; HG = high grade.

\subsection{Association of Preoperative Plasma uPAR Forms with Clinical Outcome}

The median follow-up was 34 months, range $20-55$ months. During this time $25(23 \%)$ patients experienced disease recurrence and $28(26 \%)$ patients had died, hereof $21(75 \%)$ of UCB. No patients died perioperatively.

The different uPAR forms were all significantly associated with both RFS, CSS and OS, with UPAR (I) having the strongest association-for RFS a HR of 2.26; CI (95\%): 1.45-3.50; $p=0.0003$, for CSS a HR of 2.63; CI (95\%): 1.58-4.38; $p=0.0002$, and for OS a HR of 2.56; CI (95\%): 1.64-4.00; $p=0.0001$ were demonstrated, i.e., with high concentrations predicting short survival. Additionally, we found tumour stage, lymph node status, vascular invasion, and positive resection margin statistically significantly associated with RFS, CSS, and OS, as well as a significant association between female gender and worse CSS and OS. No association was seen between clinical outcome and age, tumour grade, and neoadjuvant chemotherapy. The univariate analyses are presented in Table 3. 
Table 3. Association of the preoperative plasma uPAR forms with clinical outcome (univariate analyses).

\begin{tabular}{|c|c|c|c|c|c|c|c|c|c|}
\hline \multirow[t]{2}{*}{ Patient Characteristics } & \multicolumn{3}{|c|}{ RFS $^{\text {a }}$} & \multicolumn{3}{|c|}{$\operatorname{CSS}^{a}$} & \multicolumn{3}{|c|}{$\mathrm{OS}^{\mathrm{a}}$} \\
\hline & HR & $95 \%$ CI & $p$-Value & HR & $95 \%$ CI & $p$-Value & HR & $95 \%$ CI & $p$-Value \\
\hline \multicolumn{10}{|l|}{ uPAR levels log transformed base 2} \\
\hline uPAR (I-III) & 3.11 & $1.03-9.44$ & 0.045 & 3.41 & $1.00-11.67$ & 0.051 & 3.85 & $1.26-11.72$ & 0.018 \\
\hline uPAR (I-III) + uPAR (II-III) & 4.20 & $1.39-12.67$ & 0.011 & 4.03 & $1.37-11.87$ & 0.012 & 4.95 & $1.90-12.92$ & 0.001 \\
\hline uPAR (I) & 2.26 & $1.45-3.50$ & 0.0003 & 2.63 & $1.58-4.38$ & 0.0002 & 2.56 & $1.64-4.00$ & 0.0001 \\
\hline \multicolumn{10}{|c|}{$\begin{array}{l}\text { uPAR levels dichotomised according to } \\
\text { reference intervals } b\end{array}$} \\
\hline uPAR (I-III) & 1.90 & $0.87-4.17$ & 0.109 & 1.74 & $0.74-4.09$ & 0.207 & 1.97 & $0.93-4.20$ & 0.078 \\
\hline uPAR (I-III) + uPAR (II-III) & 2.02 & $0.89-4.56$ & 0.093 & 2.19 & $0.91-5.30$ & 0.081 & 2.93 & $1.37-6.27$ & 0.006 \\
\hline uPAR (I) & 1.62 & $0.74-3.56$ & 0.226 & 1.57 & $0.67-3.69$ & 0.303 & 1,84 & $0.86-3.91$ & 0.115 \\
\hline \multicolumn{10}{|l|}{ Clinicopathological characteristics } \\
\hline Age pr. 10 yr. age diff. & 1.00 & $0.63-1.60$ & 1.000 & 0.87 & $0.53-1.44$ & 0.594 & 0.98 & $0.62-1.54$ & 0.922 \\
\hline \multicolumn{10}{|l|}{ Gender } \\
\hline Female vs. Male & 3.60 & $1.26-8.00$ & 1.00 & 3.73 & $1.57-8.85$ & 0.003 & 2.35 & $1.05-5.24$ & 0.038 \\
\hline \multicolumn{10}{|l|}{ Tumor stage (cystectomy specimen) } \\
\hline $\mathrm{pT} \geq \mathrm{pT} 3$ pN0 vs. $\mathrm{pT} \leq \mathrm{pT} 2 \mathrm{pN} 0$ & 8.07 & $2.79-23.31$ & 0.0001 & 9.00 & $2.94-27.58$ & 0.0001 & 5.30 & $2.15-13.10$ & 0.0003 \\
\hline pTany $\mathrm{pN}+$ vs. $\mathrm{pT} \leq \mathrm{pT} 2 \mathrm{pN} 0$ & 17.51 & $6.38-48.05$ & $<0.0001$ & 11.71 & $3.08-35.93$ & $<0.0001$ & 6.32 & $2.48-16.12$ & 0.0001 \\
\hline \multicolumn{10}{|l|}{ Lymph node metastasis } \\
\hline Yes vs. No & 8.62 & $3.85-19.23$ & 0.0001 & 5.26 & $2.17-12.66$ & 0.0002 & 3.85 & $1.67-8.85$ & 0.0015 \\
\hline \multicolumn{10}{|l|}{ Pathologic grade } \\
\hline HG vs. LG & $\mathrm{NA}^{\mathrm{c}}$ & & 1.00 & $\mathrm{NA}^{\mathrm{c}}$ & & 1.00 & $\mathrm{NA}^{\mathrm{c}}$ & & 0.99 \\
\hline \multicolumn{10}{|l|}{ Vascular invasion } \\
\hline Yes vs. No & 6.99 & $3.09-15.87$ & 0.0001 & 5.20 & $2.15-12.66$ & 0.0003 & 3.86 & $1.68-8.93$ & 0.002 \\
\hline \multicolumn{10}{|l|}{ Resection margin } \\
\hline Positive vs. Negative & 6.80 & $2.54-18.18$ & 0.0001 & 5.13 & $1.72-15.38$ & 0.003 & 3.65 & $1.26-10.64$ & 0.017 \\
\hline \multicolumn{10}{|l|}{ Neoadjuvant chemotherapy } \\
\hline Yes vs. No & 0.71 & $0.27-1.89$ & 0.49 & 0.95 & $0.35-2.61$ & 0.92 & 1.41 & $0.61-3.26$ & 0.42 \\
\hline
\end{tabular}

${ }^{a}$ Analyses have been done using the Cox proportional hazards model, and the results are presented by the HR with $95 \%$ CI. The reference levels are those with an expected good prognosis, but for neoadjuvant therapy, the reference is those not receiving neoadjuvant therapy. ${ }^{b}$ Patients were dichotomized using the 95th percentile upper limit of the previous determined reference intervals for the different uPAR forms. ${ }^{c}$ NA: not accessible because of the limited number of patients with uPAR negative combined with the low event rate. $L G=$ low grade; $\mathrm{HG}=$ high grade.

\subsection{Multivariable Survival Analysis}

In the reduced multivariable survival analysis, uPAR (I) was significantly associated with CSS and OS. Tumour stage, vascular invasion, and UPAR (I-III) + uPAR (II-III) were significantly associated with RFS (Table 4). Multivariate analyses for recurrence using uPAR (I-III) + uPAR (II-III) demonstrated HR = 7.55 (95\% CI: 2.03-28.03, $p=0.003$ ), for CSS using UPAR (I) HR $=2.12$ (95\% CI: 1.28-3.51, $p=0.004)$, and for OS using uPAR(I) $\mathrm{HR}=2.11$ (95\% CI: 1.35-3.31, $p=0.001$ ). Concordance index (C-index) as a measure of discrimination was calculated for each outcome (Table 4).

Table 4. Reduced multivariate analyses.

\begin{tabular}{|c|c|c|c|c|c|c|c|c|c|}
\hline \multirow[t]{2}{*}{ Patient Characteristics } & \multicolumn{3}{|c|}{ RFS (C-Index $=0.83$ ) } & \multicolumn{3}{|c|}{ CSS (C-Index $=0.81)$} & \multicolumn{3}{|c|}{ OS (C-Index $=0.78)$} \\
\hline & HR & $95 \% \mathrm{CI}$ & $p$-Value & HR & $95 \% \mathrm{CI}$ & $p$-Value & HR & $95 \% \mathrm{CI}$ & $p$-Value \\
\hline $\begin{array}{c}\text { Clinicopathological characteristics } \\
\text { pT } \geq \text { pT3 pN0 vs. pT } \leq \text { pT2 pN0 } \\
\text { pTany pN+ vs. pT } \leq \text { pT2 pN0 } \\
\text { Vascular invasion } \\
\text { uPAR (I-III) + uPAR (II-III) } \\
\text { uPAR (I) }\end{array}$ & $\begin{array}{c}4.5 \\
19.54 \\
2.56 \\
7.55\end{array}$ & $\begin{array}{c}1.51-13.84 \\
6.00-63.61 \\
1.02-6.25 \\
2.03-28.03\end{array}$ & $\begin{array}{c}0.007 \\
<0.0001 \\
0.045 \\
0.003\end{array}$ & $\begin{array}{c}7.8 \\
11.06\end{array}$ & $\begin{array}{l}2.56-24.19 \\
3.50-34.87\end{array}$ & $\begin{array}{c}0.0003 \\
<0.0001\end{array}$ & $\begin{array}{l}4.55 \\
5.70\end{array}$ & $\begin{array}{l}1.84-11.26 \\
2.15-15.13\end{array}$ & $\begin{array}{c}0.001 \\
0.0005\end{array}$ \\
\hline
\end{tabular}

\subsection{Application of Previous Determined Reference Intervals of the Different uPAR Forms}

In a reference material consisting of interpreted healthy individuals, the 95th percentile upper limits of the reference intervals of the different uPAR forms in EDTA plasma were 
determined [19]. We know from the reference material that the UPAR levels are dependent on age and gender.

These 95th percentile upper limits were used as cut-points to dichotomise the patient cohort into patients having elevated levels of the different uPAR forms as compared to others. $36 \%$ of the UCB patients had elevated levels of UPAR (I-III), $24 \%$ had elevated levels of uPAR (I-III) + UPAR (II-III), and $27 \%$ had elevated levels of uPAR (I). As mentioned above, the actual cut-point for each marker is dependent on age and gender [19]. Univariate survival analyses demonstrated a decrease in RFS, CSS and OS in patients with levels above this cut-point of any of the uPAR forms. The strongest association was seen between uPAR (I-III) + uPAR (II-III) and OS (HR = 2.98; CI (95\%): 1.38-6.44; $p=0.0054)$ (see Table 3). Figure 2 show OS as a function of soluble uPAR (I-III) + uPAR (II-III).

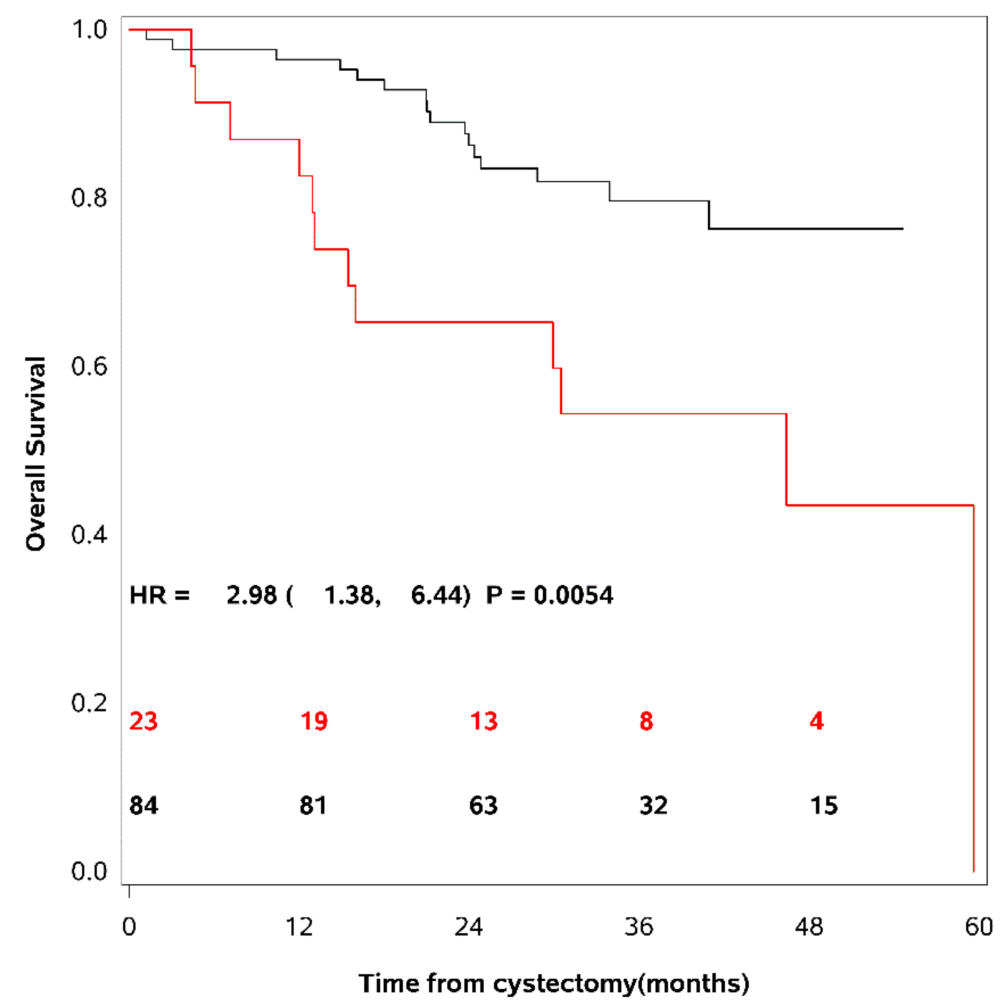

Figure 2. Overall survival as a function of soluble uPAR (I-III) + uPAR (II-III).

Multivariate analyses for recurrence using the dichotomised uPAR (I-III) + uPAR (II-III) level demonstrated HR = 3.59 (95\% CI: 1.36-9.50, $p=0.01$ ), for CSS using the dichotomised uPAR (I) level HR $=2.15$ (95\% CI: $0.81-5.69, p=0.12)$, and for OS using the dichotomised uPAR (I) level HR = 2.78 (95\% CI: 1.22-6.33, $p=0.015$ ).

Kaplan-Meier survival curves are shown using the 95th percentile of the reference intervals for dichotomisation of the patient cohort. The red line $=$ patients with uPAR above the cut-point, the black line = patients with uPAR levels below the cut-point. The number of patients at risk of death is shown for time of inclusion, 12, 24, 36, and 48 months after cystectomy. A strong association was seen between patients with levels of uPAR (I-III) + uPAR (II-III) above the cut-point and a decrease in overall survival.

\section{Discussion}

This is the first report demonstrating a significant association between high preoperative levels of the different forms of UPAR in plasma and shortened RFS, CSS and OS in patients undergoing RC for UCB. In the univariate survival analyses, the strongest association with both RFS, CSS and OS were seen for uPAR (I). In the reduced multivariate survival analysis, uPAR (I) was significantly associated with CSS and OS. Thus, the prog- 
nostic impact of uPAR (I) was independent of known prognostic factors such as tumour stage and lymph node status. This study was performed in a period where neoadjuvant chemotherapy was under implementation and had not become a routine, which explains the relatively small fraction of patients in our cohort having received this treatment. The small number of patients receiving neoadjuvant chemotherapy most likely explains that no association was seen between clinical outcome and this treatment modality.

As a control population we used the reference levels determined by Thurison et al. [19]. We chose the 95th percentile of the previous established reference intervals as cut-points to dichotomise our patient cohort. This revealed a decrease in RFS, CSS and OS in patients with levels above this cut-point of any uPAR form. In this setting, the strongest association to clinical outcome was seen for uPAR (I-III) + uPAR (II-III). Using the same approach in patients with colorectal cancer, $\mathrm{UPAR}$ (I) had the strongest discriminatory power [19]. Results may be influenced by the cut-off chosen to separate groups. These differences between cancer types could also reflect different levels of activation of the plasminogen activation system as well as of other proteases.

Interestingly, using an assay measuring the collective amounts of uPAR forms, Shariat et al. previously showed no significant impact of preoperative plasma levels of UPAR on survival in a small cohort of patients treated with RC for UCB [26]. The different methods used for quantification of the plasma uPAR levels in the study by Shariat et al. and in our study is most likely the reason for the diverse results. Shariat et al. do not present the antibodies employed in their ELISA and reveal no details on which UPAR forms their ELISA measures. We used only fully characterised monoclonal antibodies in our TR-FIAs, measuring specific uPAR forms. Cleavage of uPAR (I-III) is a sign of increased protease activity and therefore the cleaved forms, in particular UPAR (I), are stronger associated to overall survival than a mixture of the different uPAR forms, most likely measured by the ELISA used in the study by Shariat et al.

In addition to identifying the three UPAR forms in plasma and their association to clinical outcome, we demonstrated that both intact [uPAR (I-III)], and cleaved [uPAR(II-III)] forms of UPAR are present in urothelial carcinoma tissue. The purpose of this Western blot experiment was not to compare the quantities of uPAR (I-III) and uPAR (II-III) in the individual sample but to show that they are present and that the levels are higher in the tumour tissue than in the adjacent benign-appearing tissue. Because uPAR (I) does not have a glycolipid anchor, it is soluble and never found in detergent extracts of membrane proteins (tissue lysates) in contrast to uPAR (I-III) and uPAR (II-III), which is attached to the cell surface by a glycolipid anchor on domain III. UPAR (I-III) is cleaved on the cell surface by uPA in the linker region between domain I and II, liberating UPAR (I) and leaving uPAR (II-III) attached to the cell surface. We have previously published the entire blots with all the molecular weight markers (see Figures 1-3 in reference [23]. This experiment was done to ensure that cleaved UPAR might originate from the tumour tissue and not to demonstrate any correlations. This is in accordance with our previous findings in breast cancer [27].

Various sources of UPAR can contribute to the levels found in blood. It is widely accepted that increased local production of UPAR at the primary tumour site is a marker of high invasive potential which can be directly translated into poor prognosis [13,14,28,29]. Although no direct correlation between tumour tissue content and blood levels of uPAR has been reported [30], it is believed that the enhanced levels of circulating uPAR forms in cancer patients compared with healthy individuals [19] are derived predominantly from tumour tissue and accompanying stromal reactions, such as inflammation [21,31]. The concentration of the different UPAR forms in plasma is low. We have previously immunoaffinity purified soluble full-length uPAR (uPAR (I-III)) using a monoclonal antibody against an epitope on domain I [32]. This was done to visualise uPAR (I) by Western blotting in plasma from healthy individuals. We were, however, not able to visualise any uPAR (I) by Western blotting, most likely because the sensitivity of a Western blot is much lower than that of an immunoassay, including TR-FIA 4. 
The present findings and our previous findings by immunohistochemistry $[13,14]$ support the hypothesis that the tumour tissue might be the origin of the enhanced plasma levels of UPAR in patients with UCB. We did not measure the levels of the different uPAR forms in tumour tissue lysates from all patients with UCB for several reasons. The pathologist must have priority in selection of tumour tissue for diagnosis and, additionally, collection of fresh frozen tumour tissue is not standard clinical practice. Furthermore, tumour tissue is rather heterogeneous and the piece available for extraction might not be representative. Peripheral blood on the contrary is considerably more homogeneous and easier to collect than tumour tissue. Lastly, it was previously shown in breast cancer that there was no correlation between the levels found in tumour tissue and blood from the same patient [30].

Intense investigation of molecular alterations involved in different steps of invasion, progression, and metastases of UCB has revealed various promising tissue-, blood, and urine-based biomarkers as predictors for outcome in different bladder cancer settings [33-38]. Several predictive and prognostic models have been designed, but to this point none of these have reached a sufficient level of discrimination to allow implementation in daily clinical practice [37,39-43].

Owing to the complexity of the molecular abnormalities in UCB, it is unlikely that a single marker can accurately segregate tumours of similar clinicopathologic phenotypes into precise prognostic categories. To improve clinical outcome in patients with muscle invasive UCB we believe, as do other researchers, that a combination of known clinicopathological risk factors and independent, complementary molecular biomarkers might be most valuable for identification of those patients who are at highest risk of experiencing a recurrence after radical cystectomy, and therefore should be offered additional systemic therapy, or should be offered palliative treatment to avoid morbidity after radical cystectomy. The field is evolving rapidly, and the combination of possible selection markers should be based on scientific evidence, technical feasibility, and clinical relevance. At present there are, however, no suggested standard risk markers [44].

Our findings indicate that preoperative plasma levels of the cleaved uPAR forms could have a possible role in identification of patients at high risk of disease recurrence and cancer specific death which might allow a more individualised patient counselling and more focused use of perioperative therapy and/or a more individualised follow-up schedule. The patient population is, however, too small to draw firm conclusions and our data need to be validated in a larger independent patient cohort. Thus, the clinical implications are not yet clarified.

Additionally, it could be interesting to investigate the potential of soluble uPAR as a marker of recurrence during follow-up and/or as an indicator of effectiveness of ongoing treatment. If soluble uPAR is effective for these purposes, this might help to deliver more timely and relevant systemic treatment when the disease becomes advanced.

\section{Conclusions}

Our study is the first to demonstrate a significantly association between high preoperative levels of the different uPAR forms in plasma, particularly the liberated domain I- UPAR (I)—and recurrence as well as survival in patients with urothelial neoplasia undergoing radical cystectomy.

Supplementary Materials: The following are available online at https:/ /www.mdpi.com/article/10 $.3390 /$ cancers13102377/s1, Figure S1: original Western blot figure of Figure 1.

Author Contributions: Conceptualisation, L.H.D., O.D.L., M.I., G.H.-H. and H.P.; methodology, G.H.-H., S.O., I.J.C., and H.P.; software, I.J.C.; validation, L.H.D., S.O., G.H.-H. and H.P.; formal analysis, L.H.D., I.J.C.; investigation, L.H.D., P.T., L.S., H.L. and H.P.; resources, L.H.D., P.T., L.S., H.L., H.v.d.M., G.H.-H. and H.P.; data curation, L.H.D. and I.J.C.; writing—original draft preparation, L.H.D.; writing-review and editing, P.T, L.S., H.L., S.O., I.J.C., O.D.L., M.I., H.v.d.M., G.H.-H. and H.P.; visualisation, L.H.D., G.H.-H. and I.J.C.; supervision, I.J.C., H.v.d.M., G.H.-H. and H.P.; project 
administration, L.H.D., G.H.-H. and H.P.; funding acquisition, L.H.D., H.v.d.M., G.H.-H. and H.P. All authors have read and agreed to the published version of the manuscript.

Funding: This research was supported by The Oncological Research Foundation at the Department of Oncology at Rigshospitalet, Danish Cancer Research Foundation, Merchant Georg Bjørkner and wife Ellen Bjørkner Foundation, and The Harboe Foundation. Line Hammer Dohn received each award. Grant numbers and URL of websites are not available. None of the funders played any role in the study design, data collection and analysis, decision to publish, or preparation for the manuscript.

Institutional Review Board Statement: Use of the patient material was approved by The Committees of Health Research Ethics in the Capital Region of Denmark (H-1-2011-105 and H-2-2011-108) and the Danish Data Protection Agency (2007-58-0015). The study was conducted according to the guidelines of the Declaration of Helsinki and REMARK guidelines [45].

Informed Consent Statement: Informed consent was obtained from all subjects involved in the study.

Data Availability Statement: The data that support the findings of this study are available from corresponding author Line Hammer Dohn but restrictions apply to the availability of these data, which were used under license for the current study, and so are not publicly available. According to Danish law data cannot be transferred to other institutions without a data transfer agreement.

Acknowledgments: We thank Ruth Petersson for excellent technical assistance.

Conflicts of Interest: The authors declare no conflict of interest. The funders had no role in the design of the study; in the collection, analyses, or interpretation of data; in the writing of the manuscript, or in the decision to publish the results.

\section{References}

1. Babjuk, M.B.M.; Compérat, E.; Gontero, P.; Mostafid, A.H.; Palou, J.; van Rhijn, B.W.G.; Rouprêt, M.; Shariat, S.F.; Sylvester, R.; Zigeuner, R. EAU Guidelines on Non-Muscle-invasive Urothelial Carcinoma of the Bladder: Update 2016. In Proceedings of the EAU Annual Congress, Amsterdam, The Netherlands, 20-24 March 2020; ISBN 978-94-92671-07-3. Available online: http:/ / uroweb.org/guidelines / compilations-of-all-guidelines/ (accessed on 16 March 2021).

2. Witjes, J.A.B.H.; Cathomas, R.; Compérat, E.; Cowan, N.C.; Gakis, G.; Hernández, V.; Lorch, A.; Ribal, M.J.; Thalmann, G.N.; van der Heijden, A.G.; et al. European Association of Urology Guidelines on Muscle-invasive and Metastatic Bladder Cancer: Summary of the 2020 Guidelines. In Proceedings of the EAU Annual Congress, Amsterdam, The Netherlands, 20-24 March 2020; ISBN 978-94-92671-07-3. Available online: http:/ / uroweb.org/guidelines/compilations-of-all-guidelines / (accessed on 16 March 2021).

3. Hautmann, R.E.; de Petriconi, R.C.; Pfeiffer, C.; Volkmer, B.G. Radical cystectomy for urothelial carcinoma of the bladder without neoadjuvant or adjuvant therapy: Long-term results in 1100 patients. Eur. Urol. 2012, 61, 1039-1047. [CrossRef] [PubMed]

4. Stein, J.P.; Lieskovsky, G.; Cote, R.; Groshen, S.; Feng, A.; Boyd, S.; Skinner, E.; Bochner, B.; Thangathurai, D.; Mikhail, M.; et al. Radical cystectomy in the treatment of invasive bladder cancer: Long-term results in 1054 patients. J. Clin. Oncol. 2001, 19, 666-675. [CrossRef] [PubMed]

5. Yin, M.; Joshi, M.; Meijer, R.P.; Glantz, M.; Holder, S.; Harvey, H.A.; Kaag, M.; Fransen van de Putte, E.E.; Horenblas, S.; Drabick, J.J. Neoadjuvant Chemotherapy for Muscle-Invasive Bladder Cancer: A Systematic Review and Two-Step Meta-Analysis. Oncologist 2016, 21, 708-715. [CrossRef] [PubMed]

6. Svatek, R.S.; Shariat, S.F.; Novara, G.; Skinner, E.C.; Fradet, Y.; Bastian, P.J.; Kamat, A.M.; Kassouf, W.; Karakiewicz, P.I.; Fritsche, H.M.; et al. Discrepancy between clinical and pathological stage: External validation of the impact on prognosis in an international radical cystectomy cohort. BJU Int. 2011, 107, 898-904. [CrossRef]

7. Vind-Kezunovic, S.; Bouchelouche, K.; Ipsen, P.; Høyer, S.; Bell, C.; Bjerggaard Jensen, J. Detection of Lymph Node Metastasis in Patients with Bladder Cancer using Maximum Standardised Uptake Value and (18)F-fluorodeoxyglucose Positron Emission Tomography/Computed Tomography: Results from a High-volume Centre Including Long-term Follow-up. Eur. Urol. Focus 2019, 5, 90-96. [CrossRef]

8. Dano, K.; Behrendt, N.; Hoyer-Hansen, G.; Johnsen, M.; Lund, L.R.; Ploug, M.; Rømer, J. Plasminogen activation and cancer. Thromb. Haemost. 2005, 93, 676-681.

9. Hoyer-Hansen, G.; Lund, I.K. Urokinase receptor variants in tissue and body fluids. Adv. Clin. Chem. 2007, 44, 65-102.

10. Thurison, T.; Lomholt, A.F.; Rasch, M.G.; Lund, I.K.; Nielsen, H.J.; Christensen, I.J.; Hoyer-Hansen, G. A new assay for measurement of the liberated domain I of the urokinase receptor in plasma improves the prediction of survival in colorectal cancer. Clin. Chem. 2010, 56, 1636-1640. [CrossRef]

11. Almasi, C.E.; Brasso, K.; Iversen, P.; Pappot, H.; Hoyer-Hansen, G.; Danø, K.; Christensen, I.J. Prognostic and predictive value of intact and cleaved forms of the urokinase plasminogen activator receptor in metastatic prostate cancer. Prostate 2011, 71, 899-907. [CrossRef] 
12. Almasi, C.E.; Hoyer-Hansen, G.; Christensen, I.J.; Danø, K.; Pappot, H. Prognostic impact of liberated domain I of the urokinase plasminogen activator receptor in squamous cell lung cancer tissue. Lung Cancer 2005, 48, 349-355. [CrossRef] [PubMed]

13. Dohn, L.H.; Illemann, M.; Hoyer-Hansen, G.; Christensen, I.J.; Hostmark, J.; Litlekalsoy, J.; von der Maase, H.; Pappot, H.; Laerum, O.D. Urokinase-type plasminogen activator receptor (uPAR) expression is associated with T-stage and survival in urothelial carcinoma of the bladder. Urol. Oncol. 2015, 33, 165.e115-165.e124. [CrossRef]

14. Dohn, L.H.; Pappot, H.; Iversen, B.R.; Illemann, M.; Hoyer-Hansen, G.; Christensen, I.J.; Thind, P.; Salling, L.; von der Maase, H.; Laerum, O.D. uPAR Expression Pattern in Patients with Urothelial Carcinoma of the Bladder-Possible Clinical Implications. PLoS ONE 2015, 10, e0135824. [CrossRef] [PubMed]

15. Grossmann, N.C.; Schuettfort, V.M.; Pradere, B.; Moschini, M.; Quhal, F.; Mostafaei, H.; Soria, F.; Katayama, S.; Laukhtina, E.; Mori, K.; et al. Further Understanding of Urokinase Plasminogen Activator Overexpression in Urothelial Bladder Cancer Progression, Clinical Outcomes and Potential Therapeutic Targets. Onco Targets Ther. 2021, 14, 315-324. [CrossRef] [PubMed]

16. Sobin, L.H.G.M.; Wittekind, C. TNM Classification of malignant tumours. In UICC International Union Against Cancer, 7th ed.; Wiley-Blackwell: New York, NY, USA, 2009.

17. Tumours of the urinary system. In World Health Organization Classification of Tumours. Pathology and Genetics of Tumours of the Urinary System and Male Genital Organs; Eble, J.N., Sauter, G., Epstein, J.I., Sesterhenn, I.A., Eds.; IARCPress: Lyon, France, 2004; pp. 90-157.

18. Punt, C.J.; Buyse, M.; Kohne, C.H.; Hohenberger, P.; Labianca, R.; Schmoll, H.J.; Påhlman, L.; Sobrero, A.; Douillard, J.Y. Endpoints in adjuvant treatment trials: A systematic review of the literature in colon cancer and proposed definitions for future trials. J. Natl. Cancer Inst. 2007, 99, 998-1003. [CrossRef]

19. Thurison, T.; Christensen, I.J.; Lund, I.K.; Nielsen, H.J.; Hoyer-Hansen, G. Circulating intact and cleaved forms of the urokinasetype plasminogen activator receptor: Biological variation, reference intervals and clinical useful cut-points. Clin. Chim. Acta 2015, 439, 84-90. [CrossRef]

20. Piironen, T.; Laursen, B.; Pass, J.; List, K.; Gårdsvoll, H.; Ploug, M.; Danø, K.; Hoyer-Hansen, G. Specific immunoassays for detection of intact and cleaved forms of the urokinase receptor. Clin. Chem. 2004, 50, 2059-2068. [CrossRef]

21. Olson, F.J.; Thurison, T.; Ryndel, M.; Hoyer-Hansen, G.; Fagerberg, B. Soluble urokinase-type plasminogen activator receptor forms in plasma as markers of atherosclerotic plaque vulnerability. Clin. Biochem. 2010, 43, 124-130. [CrossRef]

22. Behrendt, N.; Ronne, E.; Ploug, M.; Petri, T.; Løber, D.; Nielsen, L.S.; Schleuning, W.D.; Blasi, F.; Appella, E.; Danø, K. The human receptor for urokinase plasminogen activator. NH2-terminal amino acid sequence and glycosylation variants. J. Biol. Chem. 1990, 265, 6453-6460. [CrossRef]

23. Hoyer-Hansen, G.; Ploug, M.; Behrendt, N.; Rønne, E.; Danø, K. Cell-surface acceleration of urokinase-catalyzed receptor cleavage. Eur. J. Biochem. 1997, 243, 21-26. [CrossRef] [PubMed]

24. Thurison, T.; Almholt, K.; Gardsvoll, H.; Ploug, M.; Hoyer-Hansen, G.; Lund, I.K. Urokinase receptor cleavage correlates with tumor volume in a transgenic mouse model of breast cancer. Mol. Carcinog. 2016, 55, 717-731. [CrossRef]

25. Harrell, F.E., Jr.; Lee, K.L.; Mark, D.B. Multivariable prognostic models: Issues in developing models, evaluating assumptions and adequacy, and measuring and reducing errors. Stat. Med. 1996, 15, 361-387. [CrossRef]

26. Shariat, S.F.; Monoski, M.A.; Andrews, B.; Wheeler, T.M.; Lerner, S.P.; Slawin, K.M. Association of plasma urokinase-type plasminogen activator and its receptor with clinical outcome in patients undergoing radical cystectomy for transitional cell carcinoma of the bladder. Urology 2003, 61, 1053-1058. [CrossRef]

27. Rasch, M.G.; Lund, I.K.; Almasi, C.E.; Hoyer-Hansen, G. Intact and cleaved uPAR forms: Diagnostic and prognostic value in cancer. Front. Biosci. 2008, 13, 6752-6762. [CrossRef] [PubMed]

28. Illemann, M.; Laerum, O.D.; Hasselby, J.P.; Thurison, T.; Hoyer-Hansen, G.; Nielsen, H.J.; Danish Study Group on Early Detection of Colorectal Cancer; Christensen, I.J. Urokinase-type plasminogen activator receptor (uPAR) on tumor-associated macrophages is a marker of poor prognosis in colorectal cancer. Cancer Med. 2014, 3, 855-864. [CrossRef] [PubMed]

29. Janicke, F.; Prechtl, A.; Thomssen, C.; Harbeck, N.; Meisner, C.; Untch, M.; Sweep, C.G.; Selbmann, H.K.; Graeff, H.; Schmitt, M.; et al. Randomized adjuvant chemotherapy trial in high-risk, lymph node-negative breast cancer patients identified by urokinase-type plasminogen activator and plasminogen activator inhibitor type 1. J. Natl. Cancer Inst. 2001, 93, 913-920. [CrossRef]

30. Riisbro, R.; Christensen, I.J.; Piironen, T.; Greenall, M.; Larsen, B.; Stephens, R.W.; Han, C.; Hoyer-Hansen, G.; Smith, K.; Brünner, N.; et al. Prognostic significance of soluble urokinase plasminogen activator receptor in serum and cytosol of tumor tissue from patients with primary breast cancer. Clin. Cancer Res. 2002, 8, 1132-1141.

31. Shariat, S.F.; Roehrborn, C.G.; McConnell, J.D.; Park, S.; Alam, N.; Wheeler, T.M.; Slawin, K.M. Association of the circulating levels of the urokinase system of plasminogen activation with the presence of prostate cancer and invasion, progression, and metastasis. J. Clin. Oncol. 2007, 25, 349-355. [CrossRef]

32. Hoyer-Hansen, G.; Pessara, U.; Holm, A.; Holm, A.; Pass, J.; Weidle, U.; Danø, K.; Behrendt, N. Urokinase-catalysed cleavage of the urokinase receptor requires an intact glycolipid anchor. Biochem. J. 2001, 358, 673-679. [CrossRef]

33. Kamat, A.M.; Hegarty, P.K.; Gee, J.R.; Clark, P.E.; Svatek, R.S.; Hegarty, N.; Shariat, S.F.; Xylinas, E.; Schmitz-Dräger, B.J.; Lotan, Y.; et al. ICUD-EAU International Consultation on Bladder Cancer 2012: Screening, diagnosis, and molecular markers. Eur. Urol. 2013, 63, 4-15. [CrossRef] 
34. Soria, F.; Krabbe, L.M.; Todenhofer, T.; Dobruch, J.; Mitra, A.P.; Inman, B.A.; Gust, K.M.; Lotan, Y.; Shariat, S.F. Molecular markers in bladder cancer. World J. Urol. 2019, 37, 31-40. [CrossRef]

35. Laukhtina, E.; Pradere, B.; Mori, K.; Schuettfort, V.M.; Quhal, F.; Mostafaei, H.; Sari Motlangh, R.; Katayama, S.; Grossmann, N.C.; Moschini, M.; et al. Catalog of prognostic tissue-based biomarkers in patients treated with neoadjuvant systemic therapy for urothelial carcinoma of the bladder: A systematic review. Urol. Oncol. 2021, 39, 180-190. [CrossRef] [PubMed]

36. Kamoun, A.d.R.A.; Allory, Y.; Sjödahl, G.; Robertson, A.G.; Seiler, R.; Hoadley, K.A.; Groeneveld, C.S.; Al-Ahmadie, H.; Choi, W.; Castro, M.A.A.; et al. A consensus molecular classification of muscle-invasive bladder cancer. Eur Urol. 2020, 77, 420-433. [CrossRef] [PubMed]

37. Grossman, H.B.; Bellmunt, J.; Black, P.C. Can Biomarkers Guide the Use of Neoadjuvant Chemotherapy in T2 Bladder Cancer? Eur. Urol. Oncol. 2019, 2, 597-602. [CrossRef] [PubMed]

38. Bellmunt, J.; Kim, J.; Reardon, B.; Perera-Bel, J.; Orsola, A.; Rodriguez-Vida, A.; Wankowicz, S.A.; Bowden, M.; Barletta, J.A.; Morote, J.; et al. Genomic Predictors of Good Outcome, Recurrence, or Progression in High-Grade T1 Non-Muscle-Invasive Bladder Cancer. Cancer Res. 2020, 80, 4476-4486. [CrossRef]

39. Karakiewicz, P.I.; Shariat, S.F.; Palapattu, G.S.; Gilad, A.E.; Lotan, Y.; Rogers, C.G.; Vazina, A.; Gupta, A.; Bastian, P.J.; Perrotte, P.; et al. Nomogram for predicting disease recurrence after radical cystectomy for transitional cell carcinoma of the bladder. J. Urol. 2006, 176, 1354-1361; discussion 1352-1361. [CrossRef]

40. Shariat, S.F.; Karakiewicz, P.I.; Palapattu, G.S.; Amiel, G.E.; Lotan, Y.; Rogers, C.G.; Vazina, A.; Bastian, P.J.; Gupta, A.; Sagalowsky, A.I.; et al. Nomograms provide improved accuracy for predicting survival after radical cystectomy. Clin. Cancer Res. 2006, 12, 6663-6676. [CrossRef]

41. Kluth, L.A.; Black, P.C.; Bochner, B.H.; Catto, J.; Lerner, S.P.; Stenzl, A.; Sylvester, R.; Vickers, A.J.; Xylinas, E.; Shariat, S.F. Prognostic and Prediction Tools in Bladder Cancer: A Comprehensive Review of the Literature. Eur. Urol. 2015, 68, 238-253. [CrossRef]

42. Kluth, L.A.; Xylinas, E.; Rieken, M.; Kent, M.; Ikeda, M.; Matsumoto, K.; Hagiwara, M.; Kikuchi, E.; Bing, M.T.; Gupta, A.; et al. Prognostic Model for Predicting Survival in Patients with Disease Recurrence Following Radical Cystectomy. Eur. Urol. Focus 2015, 1, 75-81. [CrossRef]

43. Kollberg, P.; Chebil, G.; Eriksson, P.; Sjödahl, G.; Liedberg, F. Molecular subtypes applied to a population-based modern cystectomy series do not predict cancer-specific survival. Urol. Oncol. 2019, 37, 791-799. [CrossRef] [PubMed]

44. Witjes, J.A.; Babjuk, M.; Bellmunt, J.; Bruins, H.M.; De Reijke, T.M.; De Santis, M.; Gillessen, S.; James, N.; Maclennan, S.; Palou, J.; et al. EAU-ESMO Consensus Statements on the Management of Advanced and Variant Bladder Cancer-An International Collaborative Multistakeholder Effort(dagger): Under the Auspices of the EAU-ESMO Guidelines Committees. Eur. Urol. 2020, 77, 223-250. [CrossRef]

45. Altman, D.G.; McShane, L.M.; Sauerbrei, W.; Taube, S.E. Reporting Recommendations for Tumor Marker Prognostic Studies (REMARK): Explanation and elaboration. PLoS Med. 2012, 9, e1001216. [CrossRef] 